ARTICLE

Received 25 Jul 2012 | Accepted 18 Mar 2013 | Published 30 Apr 2013

DOI: $10.1038 /$ ncomms2761

OPEN

\title{
A novel mechanism for fine-tuning open-state stability in a voltage-gated potassium channel
}

\author{
Stephan A. Pless ${ }^{1,2}$, Ana P. Niciforovic ${ }^{1,2}$, Jason D. Galpin ${ }^{1,2,3}$, John-Jose Nunez ${ }^{1}$, Harley T. Kurata ${ }^{1}$ \\ \& Christopher A. Ahern $1,2,3$
}

Voltage-gated potassium channels elicit membrane hyperpolarization through voltage-sensor domains that regulate the conductive status of the pore domain. To better understand the inherent basis for the open-closed equilibrium in these channels, we undertook an atomistic scan using synthetic fluorinated derivatives of aromatic residues previously implicated in the gating of Shaker potassium channels. Here we show that stepwise dispersion of the negative electrostatic surface potential of only one site, Phe481, stabilizes the channel open state. Furthermore, these data suggest that this apparent stabilization is the consequence of the amelioration of an inherently repulsive open-state interaction between the partial negative charge on the face of Phe481 and a highly co-evolved acidic side chain, Glu395, and this interaction is potentially modulated through the Tyr485 hydroxyl. We propose that the intrinsic open-state destabilization via aromatic repulsion represents a new mechanism by which ion channels, and likely other proteins, fine-tune conformational equilibria.

\footnotetext{
${ }^{1}$ Department of Anesthesiology, Pharmacology and Therapeutics, University of British Columbia, 2350 Health Science Mall, Vancouver, British Columbia, Canada V6T 1Z3. ${ }^{2}$ Department of Cellular and Physiological Sciences, University of British Columbia, 2350 Health Science Mall, Vancouver, British Columbia, Canada V6T 1Z3. ${ }^{3}$ Molecular Physiology and Biophysics, University of lowa, 285 Newton Road, lowa City, lowa 52242, USA. Correspondence and requests for materials should be addressed to C.A.A. (email: christopher-ahern@uiowa.edu).
} 
$\mathrm{V}$ oltage-gated potassium (Kv) channels are membrane proteins whose activity underlies the regulation of basal electrical excitability and action potential repolarization in virtually all electrically excitable cells ${ }^{1}$. Significant progress over the past decade in the structural biology of membrane proteins has advanced our understanding of ion channels (and especially Kv channels), likely to a much greater extent than any other membrane protein family ${ }^{2,3}$. It is now well established that these proteins are formed by four subunits, arranged symmetrically around a central, ion-conducting pore. Each subunit contains six transmembrane domains (S1-S6), with S1-S4 comprising the socalled voltage-sensor domain and S5 and S6 making up the pore domain. Under physiological conditions, $\mathrm{Kv}$ channels cycle rapidly between open and closed states and the relative stability of these states is of critical importance for a number of physiological processes ${ }^{4-6}$.

The conductive state of the pore represents the functional summation of multiple input energies, including the inherent equilibrium between open and closed conformations of the pore, as well as the 'work' that is performed by the voltage-sensing domains to bias this equilibrium. In terms of the former, computational and functional approaches support a model whereby the closed, nonconductive conformation of the pore is more stable compared with the open conformation, or conversely, the open pore represents a meta-stable complex ${ }^{7-9}$. The basis for this relative open-state instability is poorly understood, but numerous approaches have converged on the importance of side chains near the S4-S5 linker and the distal S6 segment, which line the interface between the voltage-sensing domain and cytoplasmic face of the pore region ${ }^{10-21}$. Interestingly, many of these side chains are aromatic in nature and are often highly intolerant to conventional site-directed mutagenesis.

We turned to the in vivo nonsense suppression method for incorporation of unnatural amino-acid side chains ${ }^{22}$, to subtly alter the aromatic side chains in an atom-by-atom manner, thus allowing for a more careful dissection of their physico-chemical contributions. The results show that reducing the negative surface potential on the face of a single highly conserved aromatic side chain, Phe481, through serial fluorination, leads to an increase in open-state stability, as apparent from conductance-voltage (GV) relationships, channel closing kinetics and kinetic modelling. By contrast, fluorine substitution at four nearby aromatic side chains (Phe401, Phe401, Phe484 and Tyr485) has little functional consequence. Interestingly, neutralization of a highly co-evolved nearby acidic side chain, Glu395, abolishes the effects of fluorination of Phe481, an effect not observed when neighbouring basic side chains are neutralized. Taken together, these results demonstrate for the first time the functional importance of the electrostatic surface potential of an aromatic side chain for biasing the conformational equilibria of an ion channel. Further, they are consistent with the presence of an intrinsically repulsive electrostatic interaction between the face of an aromatic and the net negative charge of a highly co-evolved acidic side chain (located on an adjacent subunit) that may serve to inherently destabilize the channel open state, an interaction potentially modulated through the Tyr485 hydroxyl moiety.

\section{Results}

Phe481 electrostatic surface potential regulates function. Structural and functional data have highlighted the importance of five aromatic side chains in the cytoplasmic end of S5 and the distal part of S6 (Fig. 1a). Importantly, four of these aromatic side chains (Phe401 and Phe402 in S5, Phe484 and Tyr485 in S6), have been suggested to have an important role in channel function $^{10-13,19}$. Further, mutations of Phe481 in S6 to nonaromatic side chains such as Ala, Asp ${ }^{23}$, Leu or Cys (Supplementary Fig. S1) result in nonfunctional channels and even the most conservative conventional mutation (Phe481Tyr) results in significant functional perturbations (Supplementary Fig. S1). Here, we sought to more clearly define the role of these five aromatic side chains in channel function, as interpretation of results obtained by conventional site-directed mutagenesis is hampered by the fact that mutations can change many physicochemical properties (such as size, hydrophobicity, H-bonding ability or charge) of a side chain. To more carefully characterize the contribution of these five aromatic side chains to pore stability, we turned to the in vivo nonsense suppression method for incorporation of unnatural amino acids ${ }^{22}$. In particular, we set out to assess the contribution of the negative electrostatic surface potential of these aromatic side chains to the inherent open versus closed equilibrium by using fluorinated Phe derivatives. These valuable tools specifically reduce the negative electrostatic surface potential of the Phe side chain, while leaving size, shape and hydrophobicity virtually unchanged. Fluorinated Phe derivatives have been extensively used to identify cation-pi interactions, a predominantly electrostatic interaction between the electronegative surface potential of an aromatic side chain and an organic cation ${ }^{24-26}$, as the addition of each fluorine leads to a stepwise decrease in the electronegative surface potential. To test if channel function is sensitive to changes in the electrostatic surface potential of Phe401, Phe402, Phe481, Phe484 or Tyr485, we incorporated $\mathrm{F}_{3}$ - $\mathrm{Phe}$ at each position, a manipulation that effectively abolishes the negative surface potential on the aromatic face. Surprisingly, trifluorination had no measurable effect on the GVs at positions 402,484 and 485 and only produced a modest $(<10 \mathrm{mV})$ right-shift in position 401 (Fig. $1 \mathrm{~b}-\mathrm{e})$. In contrast, introducing $\mathrm{F}_{3}$-Phe at position 481 led to a significant $(P<0.01)$ hyperpolarizing shift $(\sim 30 \mathrm{mV})$ in the GV (Fig. 1f, Supplementary Table S1), a particularly noteworthy phenotype because of its location at the critical interface of S6 and the S4-S5 linker ${ }^{14-21,27,28}$ and its high conservation among $\mathrm{Kv}$ channels (Fig. 2a,b). Importantly, alterations of channel gating produced by $\mathrm{F}_{3}$-Phe substitution in Phe481TAG channels was not due to nonspecific effects evoked by the in vivo nonsense suppression method as re-introducing the native Phe by nonsense suppression produced channels that were functionally indistinguishable from their wild-type (WT) counterparts (Supplementary Fig. S2, Supplementary Table S1). In contrast, replacing Phe481 by cyclohexylalanine, a cyclic but nonaromatic derivative of Phe likely resulted in nonfunctional channels, as cyclohexylalanineinjected oocytes failed to display ionic currents (Supplementary Fig. S2). These results demonstrate that a cyclic side chain alone is not sufficient for normal channel function and strengthen the hypothesis that the electrostatic surface potential of Phe 481 has a critical role in channel function.

Phe481 mediates open-state stability. Although the incorporation of $\mathrm{F}_{3}$-Phe at position 481 resulted in a marked functional phenotype, confirmation of a contribution of the negative electrostatic surface potential of the aromatic face requires demonstration of additive energetic effects of serial fluorination of the Phe side chain. This is accomplished by introduction of monoand difluorinated Phe derivatives (F-Phe, $\mathrm{F}_{2}$-Phe), which diminish the negative electrostatic surface potential of Phe 481 in a stepwise manner. As shown in Fig. 2c, each added fluorine produced a progressive left-shift in the GV and a steeper voltagedependence (Supplementary Table S1) and the free energy differences between open and closed states for WT and mutant channels $\left.\left(\mathrm{ZFV}_{1 / 2}\right)^{7}\right)$ correlated well with the number of added fluorines (Fig. 2d, Supplementary Table S1). The activation rates 
a
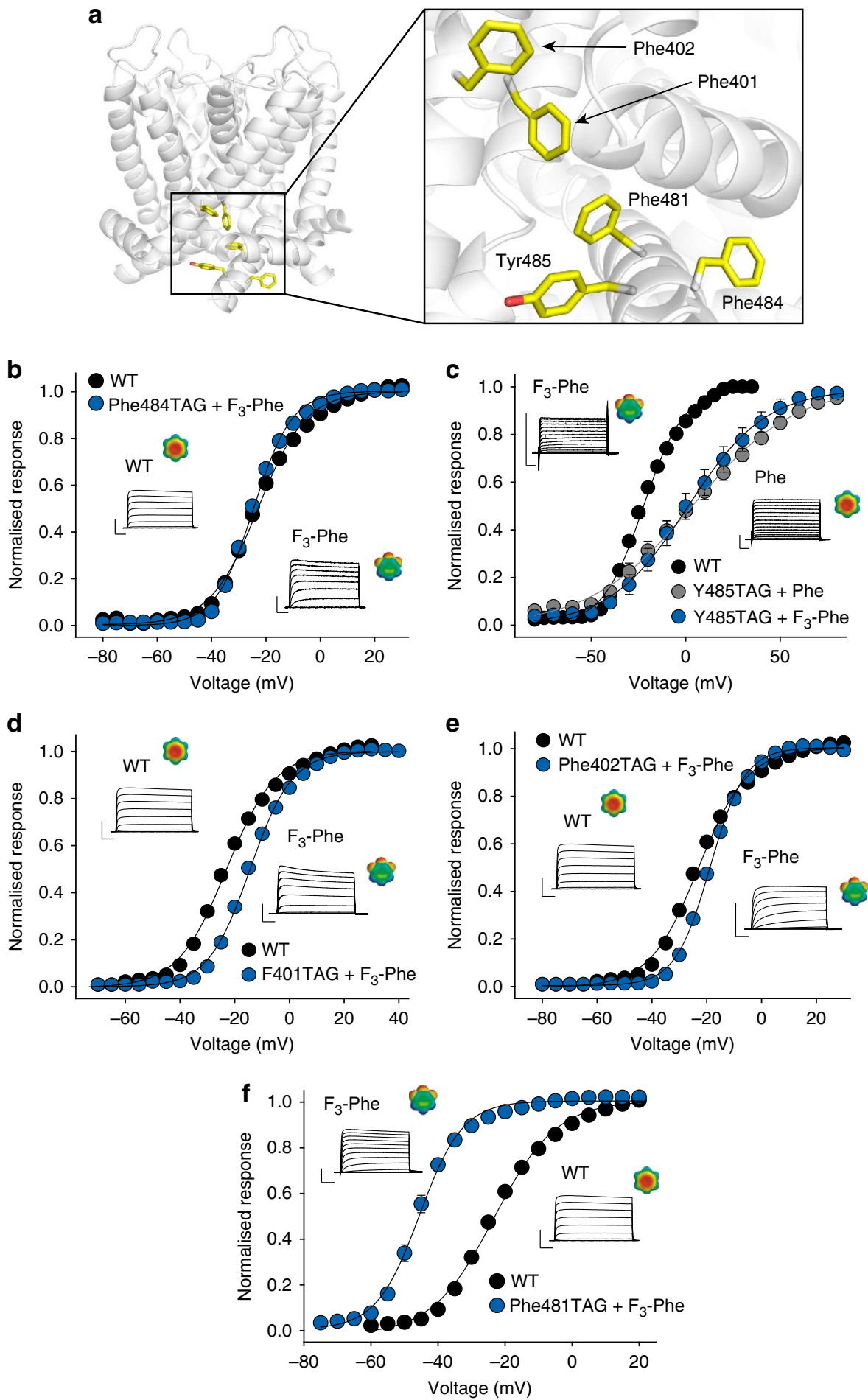

Figure 1 | The effect of fluorination is specific to position 481. (a) Model of the pore domain of the tetrameric Kv1.2/2.1 chimera (the voltage-sensing domains are omitted for clarity), with the positions of Phe401, Phe402, Phe481, Phe484 and Tyr485 highlighted in yellow. The inset shows a close-up view of the five aromatic side chains; (b-f) GVs for WT and $F_{3}$-Phe incorporated at positions 484 (b), 485 (c), 401 (d), 402 (e) and 481 (f); note that at position 485 the native Tyr was also replaced by Phe; insets show currents ( -60 to $+30 \mathrm{mV}$ in (a,c-f); -60 to $+90 \mathrm{mV}$ in (b); all in $10 \mathrm{mV}$ increments) and electrostatic surface potential maps for Phe and $F_{3}-P h e\left(E S P\right.$, red $=-15 \mathrm{kcal} \mathrm{mol}^{-1}$, green $\left.=0 \mathrm{kcal} \mathrm{mol}^{-1}, \mathrm{blue}^{-}=+15 \mathrm{kcal} \mathrm{mol}-1\right)$. Scale bar (horizontal), $20 \mathrm{~ms}$; Scale bar (vertical), $5 \mu \mathrm{A}$ in (c,d-f), $2 \mu \mathrm{A}$ in (c). WT, $\mathrm{V}_{1 / 2}=-23.0 \pm 0.5 \mathrm{mV}$; Phe401TAG $+\mathrm{F}_{3}-\mathrm{Phe}, \mathrm{V}_{1 / 2}=-14.4 \pm 0.7$; Phe402TAG + F 3 -Phe, $V_{1 / 2}=-19.0 \pm 0.6 \mathrm{mV}$, Phe484TAG $+F_{3}-$ Phe, $V_{1 / 2}=-24.6 \pm 0.6 \mathrm{mV} ;$ Tyr485TAG + Phe, $V_{1 / 2}=1.9 \pm 5.1 \mathrm{mV}$, Tyr485TAG + $F_{3}-$ Phe, $V_{1 / 2}=4.6 \pm 3.9 \mathrm{mV}$; Phe481TAG $+F_{3}-P h e, V_{1 / 2}=-45.7 \pm 0.8 \mathrm{mV}(n=4-10$ each). All data $=$ mean \pm s.e.m.

of the ionic conductance were virtually identical for all constructs (Fig. 2e), whereas the deactivation rates were slowed significantly with each added fluorine (Fig. 2f) and correlated well with the number of substituents (Supplementary Fig. S3). The fluorination-induced left-shift in the GV, concomitant with the significant slowing of the deactivation rates (and unchanged 
a

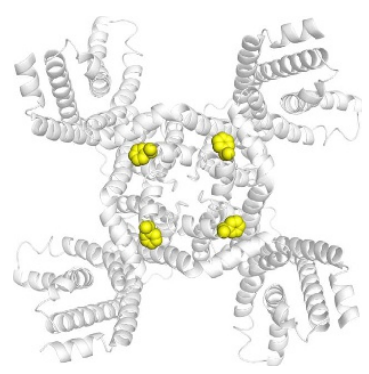

b

Shaker PVPV I VSNFNYFYHR KV1.1 PVPV I VSNFNYF YHR KV2.1 P I P I I VNNFSEFYKE KV3.1 PVPV I VNNFGMYYS L KV4.1 PVP I I V SNFSR I YHQ Kv5.1 P I HP I I NNFVRYYNK KV6.1 PVTS I FHTFSRSYLE KV7.1 PAG I LGSGFALK VQQ KV8.1 P I A I I NDR F SACYFT

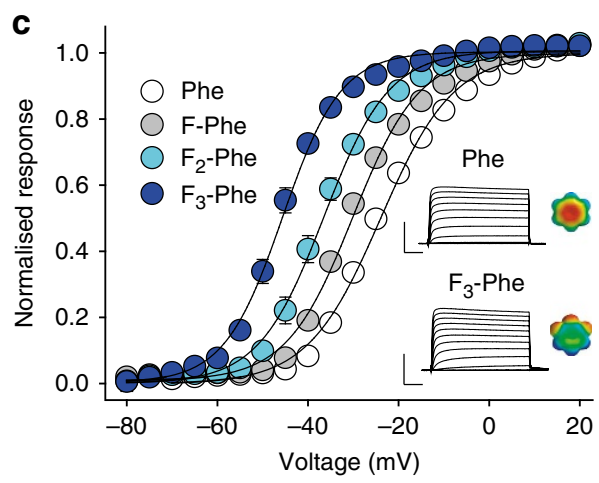

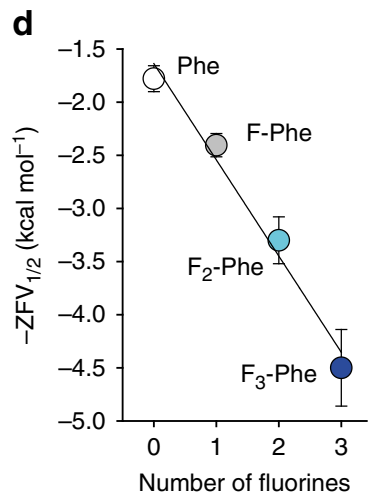
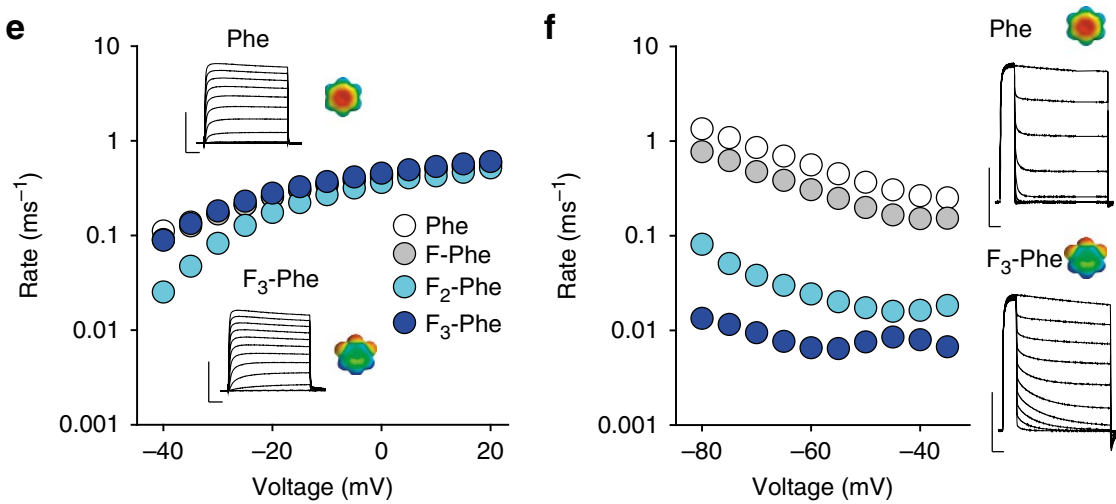

Figure 2 | Reducing the negative electrostatic surface potential on Phe481 stabilizes the channel open state. (a) Model showing the tetrameric Kv1.2/2.1 chimera from the intracellular side. The distal part of $\mathbf{S 6}$ has been removed for better visibility of Phe481 (Shaker numbering), which is highlighted in yellow. (b) Sequence alignment of the distal end of S6 of various Kv channels: Shaker (Gl:288442), Kv1.1 (Gl:119395748), Kv2.1 (Gl:84570020), Kv3.1 (Gl:298603), Kv4.1 (Gl:8272404), Kv5.1 (Gl:24418476), Kv6.1 (Gl:24418479), Kv7.1 (Gl:6166005) and Kv8.1 (Gl:7657289). The conserved Phe (Phe481 in Shaker) is highlighted in yellow. (c) GVs of Phe and fluorinated Phe derivatives incorporated at position 481 ( $n=8-10$ each; see also Supplementary Table S1); insets show representative currents ( -70 to $+40 \mathrm{mV}$ in $10 \mathrm{mV}$ increments, $F_{3}$-Phe reproduced from Fig. 1f) and electrostatic surface potential maps for Phe and $F_{3}$-Phe (scale as in Fig. 1). (d) ZFV $1 / 2$ values plotted against the number of fluorines for Phe and fluorinated Phe derivatives incorporated at position 481. (e) Activation rates plotted against voltage for Phe and fluorinated Phe derivatives incorporated at position 481; insets show sample traces for Phe and $F_{3}$-Phe (depolarizations in $10 \mathrm{mV}$ increments from a holding potential of $-80 \mathrm{mV}$, traces shown from $-70 \mathrm{mV}$ to $40 \mathrm{mV}$ ). (f) Deactivation rates plotted against voltage for Phe and fluorinated Phe derivatives incorporated at position 481; insets show sample traces for Phe and $\mathrm{F}_{3}$-Phe (after a depolarizing step to $0 \mathrm{mV}$ different test potentials were applied in $10 \mathrm{mV}$ increments, traces shown from 0 to $-100 \mathrm{mV}$ ). Scale bar (horizontal), $20 \mathrm{~ms}$ in (c,e), Scale bar, $50 \mathrm{~ms}$ in (f); Scale bar (vertical), $10 \mu \mathrm{A}$ in (c,e), Scale bar, $5 \mu \mathrm{A}$ in (f). All data = mean \pm s.e.m.

activation rates) suggested that stepwise fluorination progressively stabilizes the channel open state.

To test the validity of this hypothesis, we turned to kinetic modelling based on the Zagotta-Hoshi-Aldrich model of Shaker channel activation ${ }^{29}$ (Supplementary Fig. S4). We systematically simulated the effects of altering individual rates in the activation pathway. The simplest manipulation that recapitulates our experimental findings is to simply decelerate the closing rate for the final 'concerted' gating step (highlighted by a green box in Supplementary Fig. S4). This alteration of the model generates a leftward shift and steepening of the GV relationship, and slower deactivation rates that closely match the experimental effects of fluorination of Phe481 (Fig. 3a,b, see Supplementary Fig. S4 for specific rates and valences of model transitions). Perturbation of other model parameters failed to generate this combination of effects.

If, indeed, fluorination affected only the final opening transition, then the fluorination-induced phenotype should be retained in any mutation that separates the final opening transition from any of the earlier transitions, such as those identified by Aldrich and co-workers ${ }^{30,31}$ in the S4 segment of Shaker channels (Val369Ile, Ile372Leu, Ser376Thr or ILT). We introduced a subset of these mutations (Ile372Leu, Ser376Thr or LT) on the Phe481TAG background and introduced either Phe or
$\mathrm{F}_{3}$-Phe at position 481. As shown in Fig. 3c, simultaneous introduction of the Ile372Leu and the Ser376Thr mutation (LT) resulted in a $>80 \mathrm{mV}$ right-shift in the GV. However, trifluorination left-shifted the GV by $55 \mathrm{mV}$ towards WT and, importantly, the free energy difference between Phe and $\mathrm{F}_{3}$-Phe at position 481 was virtually identical on both the WT and the mutant (LT) background (Fig. 3d and Supplementary Table S2), strongly suggesting that fluorination does, indeed, stabilize the channel open state by altering the open-to-closed rate of the concerted pore gating step.

Effects of fluorination on S4 movement and inactivation. Despite its location in the distal part of S6, Phe481 also resides in close proximity to the S4-S5 linker and could thus directly affect electromechanical coupling and/or voltage-sensor movement. Further, although C-type (or slow) inactivation is mediated by side chains near the selectivity filter ${ }^{32}$, mutations distant from this domain have been shown to affect the process of C-type inactivation. Consequently, we set out to test directly if fluorination of Phe481 has effects on S4 movement and/or C-type inactivation. To address the former, we used voltageclamp fluorometry (VCF) $)^{33,34}$ to track S4 movement with a fluorophore as a reporter. We found that labelling Ala359Cys 

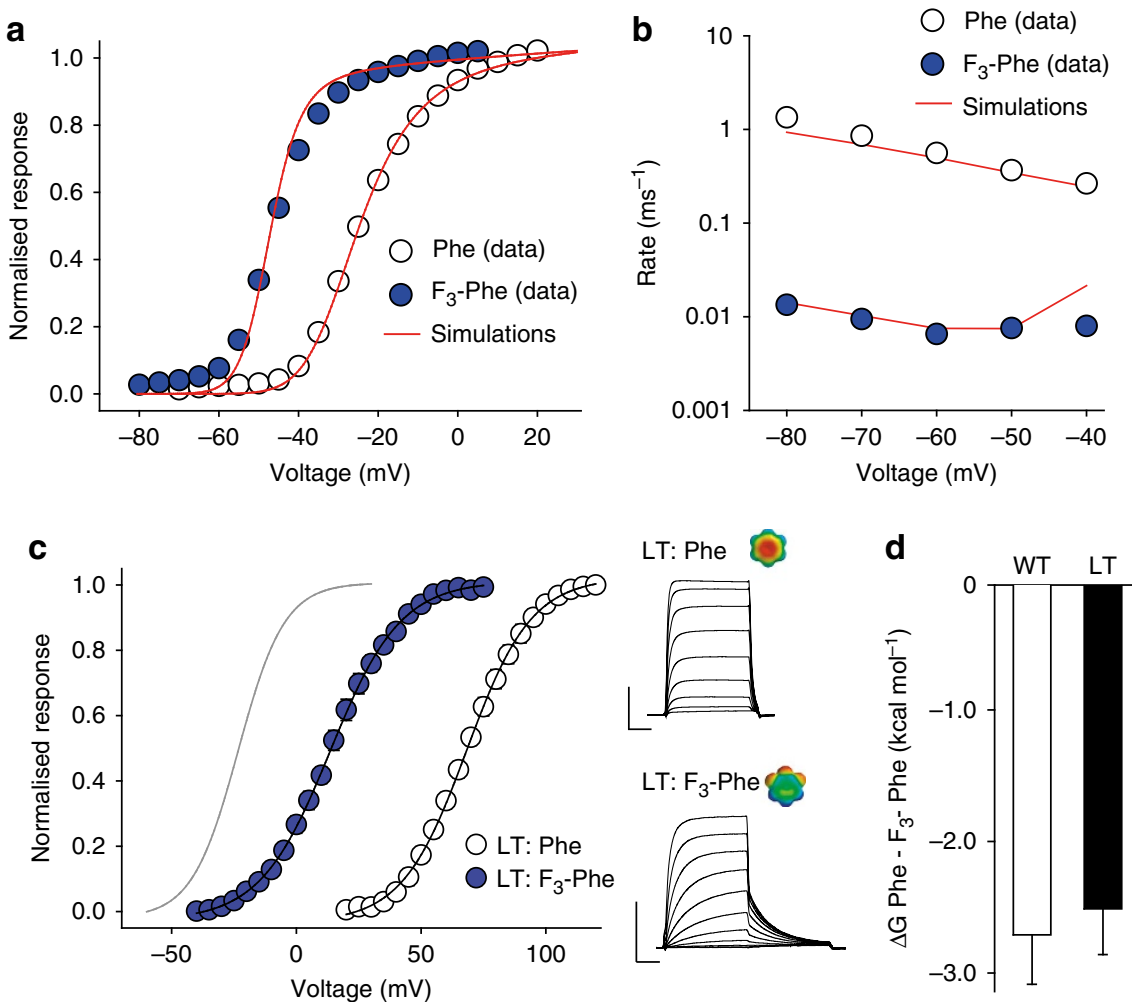

Figure 3 | Kinetic modeling and mutational studies confirm open-state stabilization through fluorination. (a) Experimentally observed GV data points for Phe and $\mathrm{F}_{3}$-Phe (open and blue circles, respectively; reproduced from Fig. 1f) overlaid by the simulated data based on kinetic modelling using the ZHA model (red lines); (b) Experimentally observed deactivation rates for Phe and $\mathrm{F}_{3}$-Phe (open and blue circles, respectively; reproduced from Fig. 2f) compared with the simulated deactivation rates for Phe and $F_{3}$-Phe based on kinetic modelling using the ZHA model (red lines); (c) GVs for Phe and $F_{3}$-Phe introduced on the lle372Leu, Ser376Thr (LT) double-mutant background (open and blue circles, respectively). The fit for the WT GV is shown in grey, insets show representative currents. Scale bar (horizontal), $20 \mathrm{~ms}$; Scale bar (vertical), $4 \mu \mathrm{A}$. (d) Bar diagram comparing the free energy difference between Phe and $F_{3}$-Phe incorporated at position 481 on the WT (empty bar) or LT double-mutant background (black bar). All experimental data $=$ mean \pm s.e.m.

(located at the top of S4) with tetramethylrhodamine-5maleimide with either $\mathrm{Phe}$ or $\mathrm{F}_{3}$-Phe in position 481 resulted in robust fluorescence signals that displayed an identical voltage-dependence (Supplementary Fig. S5), suggesting that fluorination of Phe 481 specifically alters the inherent open versus closed equilibrium of the pore domain but not the voltagedependence of S4 movement. Next, we set out to investigate the possibility of functional coupling between Phe 481 and the selectivity filter region. However, comparing the time course and extent of C-type inactivation with either Phe or $\mathrm{F}_{3}$-Phe in position 481 yielded identical results (Supplementary Fig. S6), demonstrating that fluorination does not affect C-type inactivation.

Identifying potential interaction partners of Phe481. The functional impact of fluorination is highly specific for position 481 and originates from changes in the electrostatic surface potential. To identify possible interaction partners that may be involved in this electrostatic effect, we turned to statistical coupling analysis (SCA $)^{35,36}$, a method for identification of co-evolved pairs of side chains (or domains) ${ }^{37}$ (Supplementary Fig. S7). In particular, we focused on charged side chains within $13 \AA$ of Phe481: four basic side chains (Lys380, Arg387, Lys390 and Arg394) and a single acidic side chain (Glu395). In effect, we sought to determine whether Phe481 engages in an energetically attractive (cation-pi) interaction with a nearby basic side chain or in an energetically repulsive interaction mediated by an acidic side chain. Figure 4a shows a pseudo-heat map of SCA scores for Phe481 and all five charged side chains, illustrating the relatively low values for the basic residues, and surprisingly high coevolution scores for Phe481 and Glu395 when compared against the entire channel sequence. We generated a histogram illustrating the frequency of SCA scores among all pairwise comparisons in the channel, highlighting the SCA scores of all five charged side chains (Lys380, Arg387, Lys390, Arg394 and Glu395), with Phe481 (Fig. 4b). This analysis yields a remarkable disparity between the four basic side chains and the single acidic side chain: while Arg387, Lys380, Arg394 and Lys390 all had low scores $\left(0.13 \mathrm{kT}^{\star}, 0.20 \mathrm{kT}^{\star}, 0.21 \mathrm{kT}^{\star}\right.$ and $0.47 \mathrm{kT}^{\star}$, respectively, in terms of 'statistical energy'), Glu395 had one of the highest scores in the entire data set $\left(1.93 \mathrm{kT}^{\star}\right)$ (Fig. $4 \mathrm{~b}$ ), indicating a particularly high degree of co-evolution between Phe481 and Glu395, an observation further highlighted by the close physical proximity of the two side chains (see structural model in Fig. 4b). Additional evidence for a potential interaction between Phe481 and Glu395 stems from the observation that in Kv sequences with a Phe in the position equivalent to Shaker 481, only the position equivalent to Glu395 shows a strong preference for a single, acidic side chain (Glu), while positions 380, 387, 390 and 394 show little or no side chain preference (Supplementary Fig. S8).

Phe481 does not interact with nearby basic side chains. Our functional data show that Phe481 is likely engaged in an electrostatic interaction, and SCA suggests that four nearby basic side 

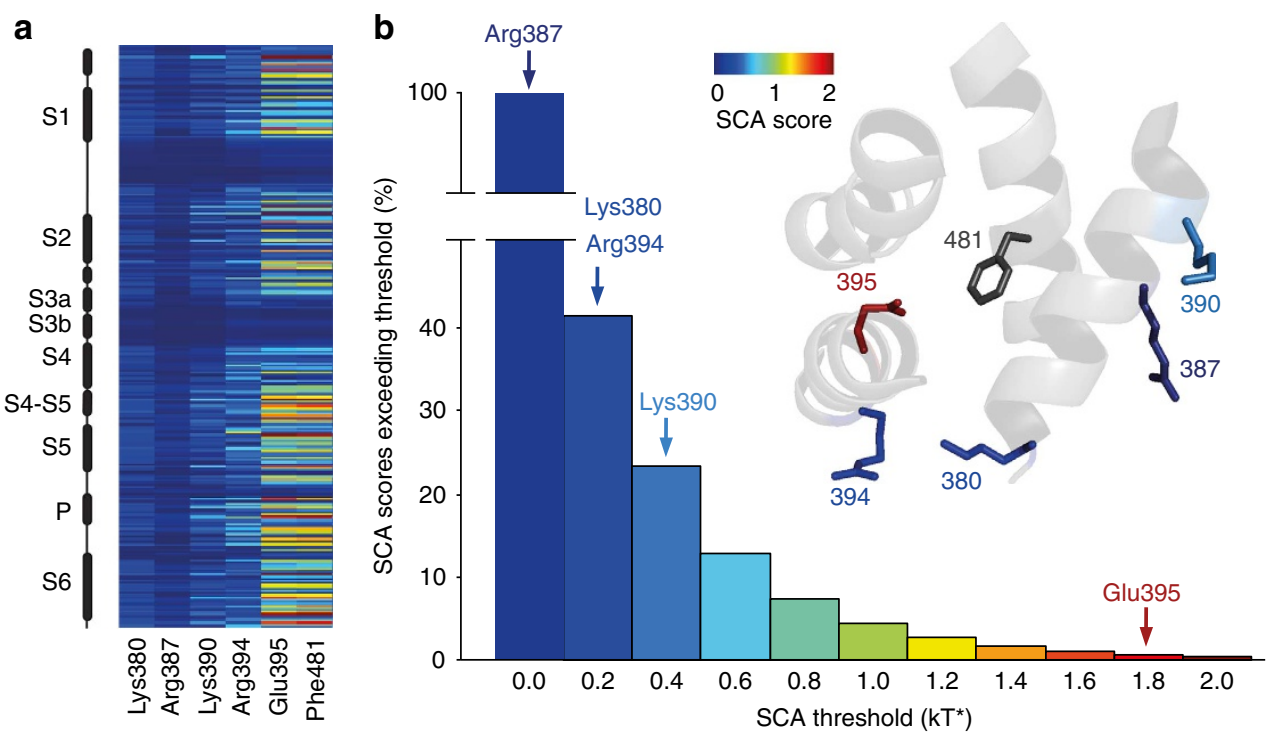

Figure 4 | SCA analysis highlights the varying levels of co-evolution between Phe481 and nearby charged side chains. (a) SCA scores of Phe481 and all five charged side chains that are within $12 \AA$ of Phe 481 with the vertical axis representing all side chains considered for the SCA analysis (corresponding to positions 208 to 487 in Shaker, with black boxes representing $\alpha$-helical regions in the channel). Scale bar as in (b). (b) Bar diagram indicating the percentage of SCA scores exceeding the binned threshold indicated on the $x$ axis for all pairings in the SCA analysis. The SCA scores of all five charged side chains with respect to Phe481 are highlighted with arrows (Arg387 $=0.13 \mathrm{kT} T^{\star}$, Lys380 $=0.20 \mathrm{kT}{ }^{\star}, \operatorname{Arg} 394=0.21 \mathrm{kT}{ }^{\star}, \mathrm{Lys} 390=0.47 \mathrm{kT} T^{\star}$ and Glu395 =1.93 kT*); Note the high co-evolution score for the Glu395-Phe481 coupling. Inset shows the scale of the heat map used in (a,b) and a model displaying all five charged side chains that are within $13 \AA$ of Phe481. Note that the charged side chains are coloured by their SCA score values (Phe481 is shown in dark grey as all SCA scores are relative to Phe481 in this analysis).

chains are improbable candidates for a (co-evolved) interaction with Phe481. To validate our statistical approach we reasoned that neutralizing any of the four basic side chains should have no impact on the fluorination-induced phenotype at Phe481. Indeed, we found that neutralization of Lys380, Arg387, Lys390 or Arg394 had no effect on the fluorination of Phe481: we still observed a stepwise left-shift in the GV with fluorination (Fig. 5a-d) and plotting the respective $\Delta \mathrm{ZFV}_{1 / 2}$ values against the number of fluorines resulted in a linear trend, comparable to that obtained with the WT background (Fig. 5f, Supplementary Table S1). Additionally, we investigated a possible role of Arg377 because despite its large ( $>18 \AA$ ) distance to Phe481, Arg377, similar to Phe481, is highly sensitive to modest conventional mutations ${ }^{38,39}$. The charge-neutralizing mutation Arg377Gln is nonfunctional ${ }^{38,39}$, but if a specific electrostatic interaction between Arg377 and Phe481 was present, even replacing Arg by Lys should result in altered interaction energetics ${ }^{40,41}$. However, the resulting free energy difference on the Arg377Lys background was identical to that of WT, ruling out an electrostatic interaction between Arg377 and Phe481 (Fig. 5e,f).

Neutralizing Glu 395 abolishes the effect of fluorination. If the electrostatic surface potential of Phe 481 is crucial for open-state stability, but nearby positively charged side chains do not appear to have a role, what other side chains could be involved in the electrostatic interaction with Phe481? Interestingly, Glu395 is not only highly conserved among Kv channels (Fig. 6a), it is also the only other charged side chain in the vicinity of Phe481 (around $7 \AA$ away in the Kv1.2/Kv2.1 chimera open channel crystal structure $^{3}$ and around $5 \AA$ away in a refined model of the Kv1.2 crystal structure ${ }^{42}$ ) (Fig. 6b). More importantly, our SCA analysis identified Glu395 as the candidate with the highest co-evolution score of all charged residues within $13 \AA$ of Phe481 (see Fig. 4). Figure $6 \mathrm{c}$ shows the lack of a stepwise left-shift in the GV through fluorination at position 481 on the Glu395Gln background, exemplified by the incorporation of $\mathrm{F}_{3}$-Phe, which results in a GV that is indistinguishable from that of Phe incorporated in position 481 on the Glu395Gln background (Supplementary Table S1). The effect is made clear by plotting the free energies of these three mutants, as well as mutants obtained through fluorination on a different hydrophilic background (Glu395Asn, Fig. 6d,e) and various hydrophobic backgrounds (Glu395Ala, Glu395Leu and Glu395Ile, Supplementary Fig. S9 and Supplementary Table S1), none of which show a trend with fluorination.

A possible role for Tyr485 in modulating the interaction. Given that some structural data ${ }^{3}$ suggest the Tyr hydroxyl of Tyr 485 to reside between Phe481 and Glu395, we tested the effect of fluorination in position 481 on the Tyr485Phe background to examine a potential modulating role of the Tyr hydroxyl in the repulsive interaction between Phe481 and Glu395. Removal of the Tyr485 hydroxyl resulted in a large right-shift of the GV, as well as a rapid and irreversible inactivation phenotype (Supplementary Table S1 and Supplementary Fig. S10); and fluorination in position 481 did have no further effect on channel function, potentially suggesting that the Tyr485 hydroxyl modulates an indirect repulsive interaction between Phe481 and Glu395. However, the drastic phenotype of Tyr485Phe alone could be indicative of major structural perturbations that may result in a nonspecific disruption of the interaction between Phe 481 and Glu395, thus complicating the interpretation of the findings with Tyr485Phe.

Further insight into a potential repulsive interaction. Given that our findings point towards an important role of the negative electrostatic surface potential of Phe481 and statistical, structural and functional data support the notion of Glu395 being a potential (direct or indirect) interaction partner, we turned to $a b$ 

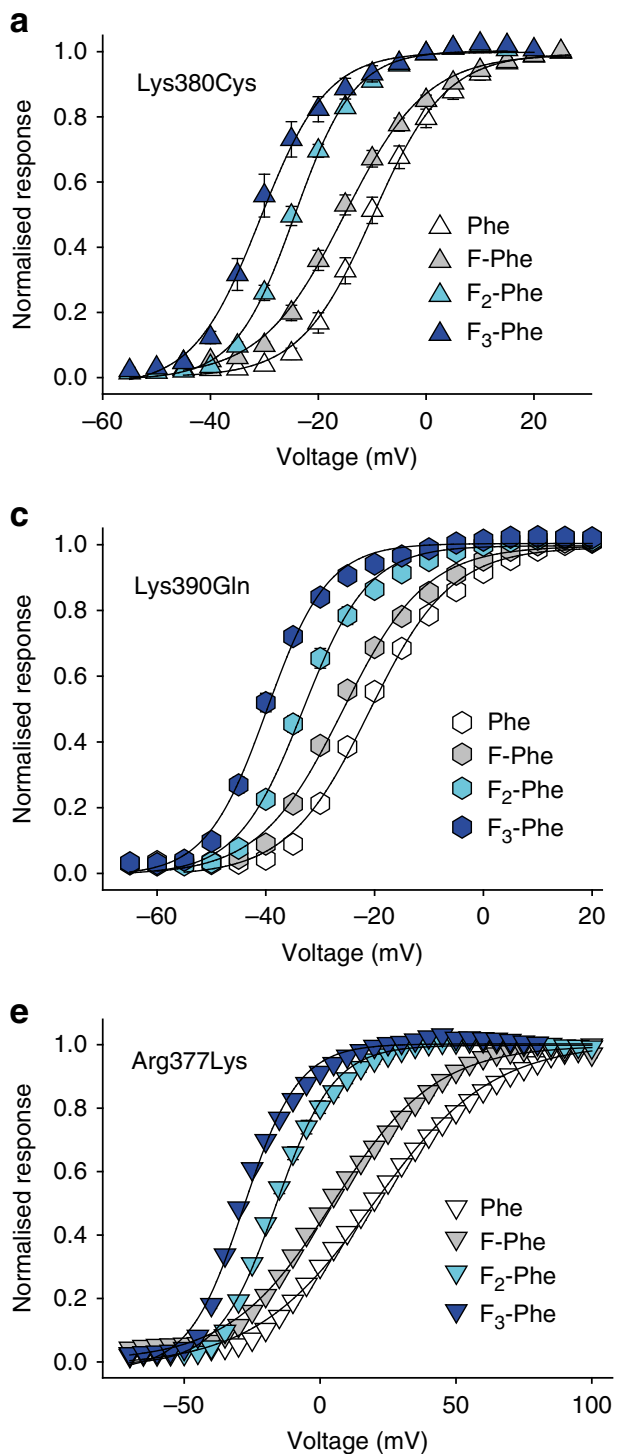
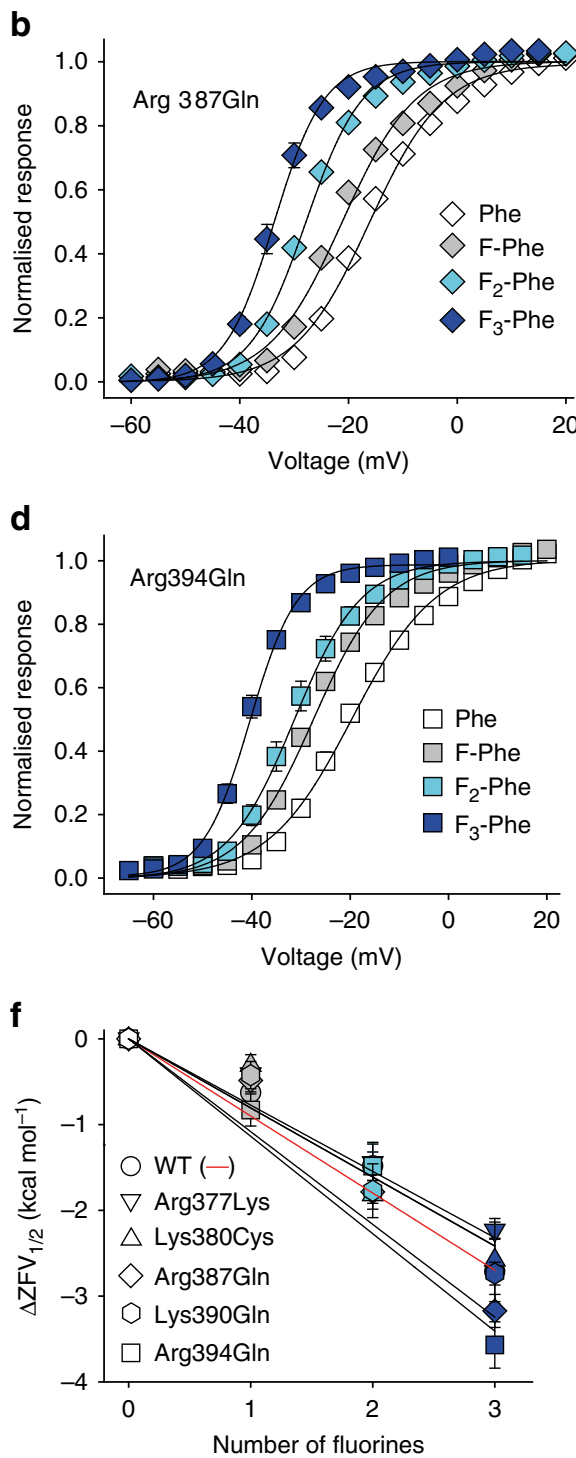

Figure 5 | Nearby positive charges are unlikely to interact with the electrostatic surface potential of Phe481. (a-e) GVs of Phe and fluorinated Phe derivatives incorporated at position 481 on the background of Lys380Cys (a), Arg387Gln (b), Lys390Gln (c), Arg394Gln (d) and Arg377Lys (e) $\left(n=3-11\right.$ each, see also Supplementary Table S1); (f) $\Delta Z F_{1 / 2}$ values plotted against the number of fluorines for Phe and fluorinated Phe derivatives incorporated at position 481 on the background of Lys380Cys, Arg387Gln, Lys390Gln, Arg394Gln and Arg377Lys. Lines indicate linear fits obtained with $f=y 0+a^{\star} x$, with $y 0=0$ (mutants in black, WT in red). All data $=$ mean \pm s.e.m.

initio calculations to further characterize such a possible repulsive electrostatic interaction. First, we aligned a negatively charged acetate ion with the face of a Phe and calculated the resulting energy over a range of distances. Not surprisingly, the energy required to maintain a given distance rapidly dissipates with increasing separation (Supplementary Fig. S11). Plotting the experimentally determined free energy differences based on the fluorination of Phe 481 against the predicted energies at an acetate-Phe distance of $7 \AA$ (similar to that observed in the Kv1.2/ Kv2.1 chimera open channel crystal structure ${ }^{3}$ ), we observed a very strong inverse correlation $(R=0.98)$, with a slope of $(-) 1.0$ (Supplementary Fig. 11). However, it should be noted that the (unknown) dielectric constant in the space between Phe481 and Glu395 would modulate the strength of the interaction, complicating a direct conclusion about the precise distance. Finally, we sought to test two possible relative orientations of the aromatic ring and the carboxyl group by calculating the interaction energies between a carboxyl group and Phe or fluorinated Phe derivatives in both an en face and an en edge orientation (Supplementary Fig. 11). Although the orientation of Phe481 and Glu395 varies among different structural and computational models $3,42,43$, the results from the $a b$ initio calculations suggest that the experimentally observed data are consistent with an en face orientation, as the data for the en edge orientation predicts large positive and non-linear energy changes with fluorination, in contrast to the linear and negative changes observed experimentally. It should be noted that in principle, Phe481 could adopt different rotamer conformations, but while we cannot rule out this possibility, it seems unlikely given the limited space around Phe481 (due to the close proximity of Tyr485, Phe402, Thr388 and Leu472).

\section{Discussion}

Our results show that Phe481, located at the distal end of S6, is unique among the numerous aromatic side chains found in this 
a

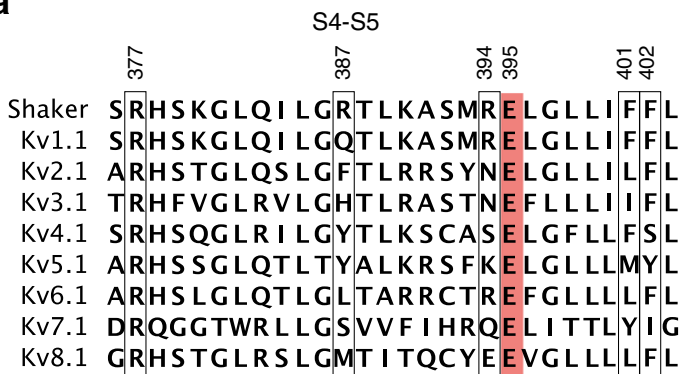

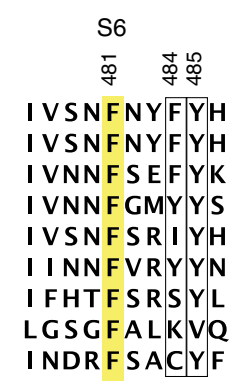

b

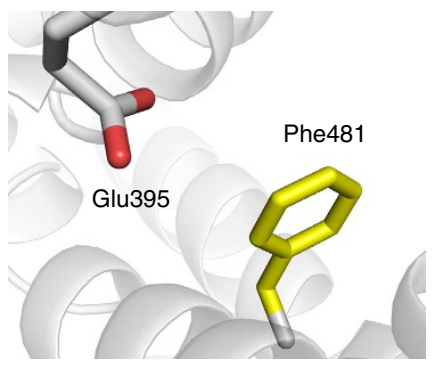

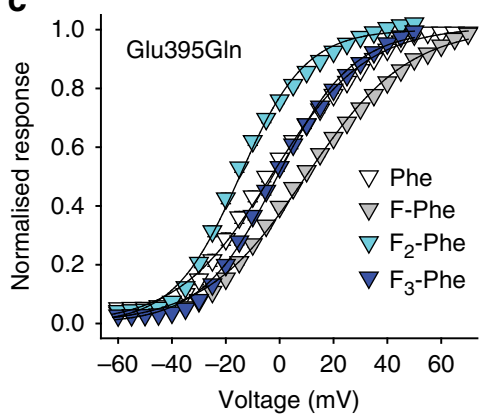

d

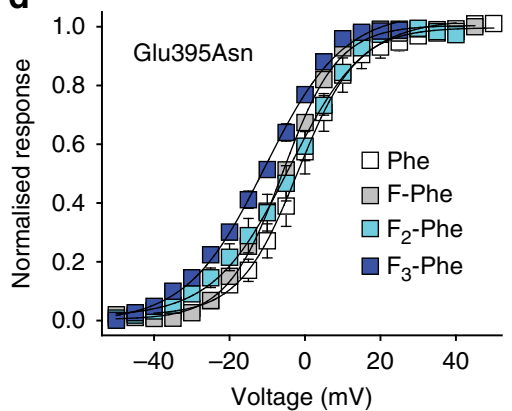

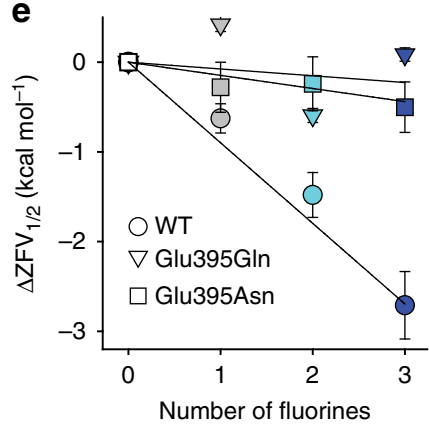

Figure 6 | Glu395 is likely to inherently destabilize the open state via electrostatic repulsion with Phe481. (a) Sequence alignment of the S4-S5 linker and the distal part of S6 in various Kv channels (see Fig. 1 for details). The conserved Glu and Phe side chains are highlighted in red and yellow, respectively; other residues tested in this study are highlighted by black boxes (Shaker numbering); (b) Model highlighting the proximity of Glu395 to Phe481 (based on a refined model of the Kv1.2 open channel crystal structure ${ }^{42}$ ); (c,d) GVs of Phe and fluorinated Phe derivatives incorporated at position 481 on the background of Glu395GIn (c) and Glu395Asn (d) ( $n=3-6$ each; see also Supplementary Table S1); (e) $\Delta$ ZFV $1 / 2$ values plotted against the number of fluorines for Phe and fluorinated Phe derivatives incorporated at position 481 on the background of Glu395GIn and Glu395Asn (compared with WT). Lines indicate linear fits obtained with $f=y 0+a^{\star} x$, with $y 0=0$. All data $=$ mean \pm s.e.m.

area, in that fluorination induces a highly specific stabilization of the open state. The phenotype elicited through fluorination suggests that the negative electrostatic surface potential on the face of Phe481 is crucial for the observed effect. Furthermore, we used SCA to identify potential charged interaction partners near Phe481. Although four basic side chains were found to be close to Phe481, all displayed low SCA values and none altered the effect of fluorination at position 481 when neutralized. In contrast, neutralization of Glu395, a highly co-evolved acidic side in very close proximity to Phe481 (although located on the adjacent subunit), abolished the effects of fluorination at Phe481, raising the possibility of a repulsive interaction between the face of Phe481 and the carboxyl moiety of Glu395 in the channel open state (Fig. 7). This notion is not only consistent with the unusually high coupling score for the Phe481-Glu395 pair but further supported by $a b$ initio calculations. It should be noted, however, that our data do not fully exclude the possibility of an indirect interaction between Phe481 and Glu395, such as would arise from another side chain located between Phe481 and Glu395 (with Tyr485 being a potential candidate, see Supplementary Fig. S10), a lipid head group located between the two side chains (an acyl chain is located within about $8 \AA$ of Phe 481 in the Kv1.2/Kv2.1 chimera open channel crystal structure, although no head group was resolved $^{3}$ ) or an indirect steric effect/local rearrangement caused by mutations at position 395 .

Our finding that Phe 481 is highly sensitive to replacement with both naturally occurring and unnatural side chains is entirely consistent with previous studies on Shaker ${ }^{12,44}$ and other Kv channels ${ }^{15,20}$, which have shown that conventional mutations have potent functional effects and often result in nonfunctional channels. Further, it is noteworthy that the Phe481 equivalent aromatic side chain is absolutely conserved among all sodium
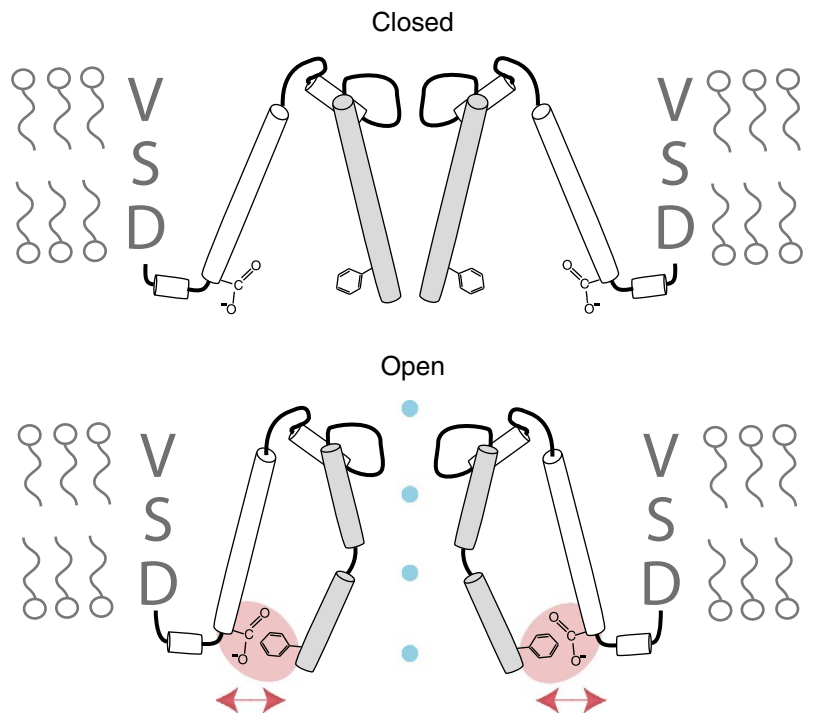

Figure 7 | Cartoon of a putative repulsive electrostatic interaction between Phe481 and Glu395 in the channel open state. Cartoon of the pore forming helices of two opposing potassium channel subunits in the closed (upper panel) and open (lower panel) conformation, respectively. VSD stands for voltage-sensor domain. The close proximity of the Glu395 carboxyl and the Phe481 aromatic moiety in the open state are highlighted by the red oval; the electrostatic repulsion between the two is symbolized by the red arrows. The blue dots represent potassium ions along the permeation pathway. Note that for reasons of simplicity the model depicts an intrasubunit interaction between Glu395 and Phe481, while in the fulllength channel the shortest distance between Glu395 and Phe481 is between adjacent subunits, thus indicating an intersubunit interaction. 
channel isoforms ${ }^{45}$. Interestingly, an erythromelalgia-associated Val substitution of the equivalent Phe in domain III of the sodium channel isoform 1.7 (Nav1.7) also results in a pronounced left-shift in the GV (and virtually no effect on activation kinetics) ${ }^{45,46}$. These data may suggest a stabilization of the channel open state similar to that observed with fluorinated Phe derivatives at position 481 in Shaker. Reminiscent of studies highlighting the critical importance of Phe481, Glu395 has also been implicated previously as a determinant of pore stability: substitutions with the small, hydrophobic side chain Ala drastically left-shift the GV, similar to effects of trifluorination at Phe481 (Supplementary Table S1) 7,19 , while replacement with Trp causes an extreme $(>200 \mathrm{mV})$ right-shift in the $\mathrm{GV}^{11}$, suggesting that charge is not the only critical parameter for channel function at position 395 (consistent with the observation that the Glu395Asp mutation causes drastic functional consequences and is a possible cause of episodic ataxia ${ }^{47}$ ). Finally, structural studies have demonstrated the close physical proximity of the equivalent side chains to Phe481 and Glu395 in Kv channel $^{3,42}$ and, more importantly, comparison of open and closed structures of the full-length prokaryotic KcsA potassium channel show that the equivalent side chains to Phe481 and Glu395 approach each other by about $2 \AA$ in the channel open state, coming as close as $4 \AA$ (refs 48,49), a notion further supported by a recent all-atom simulation of $\mathrm{Kv}$ channel gating transitions ${ }^{9}$.

It should be noted that aromatic molecules possess the unique capability to engage in attractive interactions with both cations (cation-pi interactions ${ }^{24,25}$ ) and anions (anion-pi interactions $\left.s^{44,50}\right)$. However, in the present study, both of these possibilities seem very unlikely: if Phe481 was involved in a cation-pi interaction, fluorination would abolish this interaction and thus would not manifest as a stabilizing a certain state (rather, fluorination would destabilize a particular state, a phenotype that is not observed experimentally). Anion-pi interactions are also attractive in nature and result from heavily substituted aromatic systems to generate a positive quadrupole moment sufficient in strength to engage in attractive interactions with anions. These conditions are clearly not fulfilled by $\mathrm{F}_{3}-\mathrm{Phe}$ and its quadrupole moment near zero ${ }^{50,51}$.

Is it possible that the dipole elicited through fluorination is responsible for the drastic stabilization of the channel open state? We believe this is unlikely for four reasons. First, the F-Phe and $\mathrm{F}_{2}$-Phe side chains both have dipole moments of around 1.9 debye and should thus result in an identical functional effect on the channel, which is not seen experimentally. Second, if the altered dipole of a fluorinated Phe derivative is responsible for the openstate stabilization, then mutations of other residues would be unlikely to eliminate the effect of fluorination at position 481 . However, neutralizing Glu395 effectively abolished the effect of fluorination at Phe481. Third, the dipole moment of the Tyr side chain is comparable to that of F-Phe and $\mathrm{F}_{2}$-Phe (1.7 debye versus 1.9 debye) but the functional effects vary drastically: although Tyr in position 481 leads to a dramatic $(100 \mathrm{mV})$ right-shift in the GV, F-Phe and $F_{2}$-Phe both left-shift the GV by 6 and $12 \mathrm{mV}$, respectively. Lastly, $a b$ initio calculations, which factor in effects of fluorination on the aromatic dipole, show that an en edge interaction predicts an energetically non-linear effect, which also was not borne out in the functional consequence of fluorination at Phe481. Similarly, it is improbable that the observed effect of open-state stabilization is caused by a steric effect of the fluorinated Phe derivatives because the addition of a single fluorine adds $5 \AA^{3}$ to the volume of a Phe side chain, while introducing a hydroxyl group adds $8 \AA^{3}$ (www. molinspiration.com) but both have opposing functional effects (see above).
Taken together, the data demonstrate that the electrostatic surface potential of a highly conserved Phe 481 regulates openstate stability in Shaker channels and further support the notion of a repulsive interaction between Phe 481 and Glu395, which could serve to inherently destabilize the channel open state in WT channels. Regardless of whether the interaction between Phe481 and Glu395 is direct or indirect (modulated possibly through the Tyr485 hydroxyl), we speculate that upon channel opening, the negatively charged carboxyl moiety of Glu395 and the electronegative surface potential on the face of Phe 481 come in closer proximity than in the closed state and thus lead to an electrostatic repulsion that modulates open-state stability. Although rare, glutamate residues buried in hydrophobic pockets have been reported befor ${ }^{52-54}$ and the resulting energetic penalty has been suggested to be important for protein function ${ }^{53,55}$. The proposed mechanism of state-dependent electrostatic repulsion could be functionally relevant in other ion channel types, and may be a broadly employed energetic contribution in balancing conformational dynamics in other protein types as part of the general tradeoff between stability and function ${ }^{56}$.

\section{Methods}

Molecular biology and in vivo nonsense suppression. Residues 6-46 were removed from Shaker cDNA (in pBSTA) to abolish N-type inactivation. All constructs contained the point mutations C301S, C308S and T449V (unless stated otherwise) and successful incorporation of these and all additional mutations was confirmed by automated sequencing. cRNA was transcribed from template cDNA with the mMessage mMachine kit (Ambion, Austin, TX, USA). Stage V-VI Xenopus laevis oocytes were prepared, and injected as previously described ${ }^{57}$ Oocytes were incubated for $8-48 \mathrm{~h}$ after injection (at $18^{\circ} \mathrm{C}$ ). Fluorinated Phe derivatives (4-F-Phe (F-Phe); 3,5- $\mathrm{F}_{2}-\mathrm{Phe}\left(\mathrm{F}_{2}-\mathrm{Phe}\right)$ and $3,4,5-\mathrm{F}_{3}-\mathrm{Phe}\left(\mathrm{F}_{3}-\mathrm{Phe}\right)$ ) were purchased from Asis Chem (Watertown, MA) and Sigma Aldrich (St Louis, MO). The in vivo nonsense suppression methodology has been described in detail elsewhere ${ }^{22}$. In brief, unnatural amino acids (aa) were protected with nitroveratryloxycarbonyl and were subsequently activated as the cyanomethyl ester. The resulting product was then coupled to the dinucleotide dCA (Dharmacon, Lafayette, CO) and ligated to a modified (G73) Tetrahymena thermophila tRNA. Immediately before co-injection with the channel cRNA, UV irradiation was used to deprotect the aminoacylated tRNA-aa from the nitroveratryloxycarbonyl protection group. Typically, 10-80 ng of tRNA-aa and 25-50 ng of channel cRNA were injected in a $50 \mathrm{nl}$ volume. To test for nonspecific incorporation of endogenous amino acids and/or recharging of the injected tRNA, the cRNA together with a tRNA coupled to pdCpA (without an appended aa) were injected. Even at large depolarizations $(+50 \mathrm{mV})$ currents for Phe401TAG, Phe402AG, Phe481TAG, Phe484TAG and Tyr485TAG constructs were never larger than those recorded from oocytes injected with water, $(<500 \mathrm{nA}, n=4$ each).

Electrophysiology and fluorometry. Two electrode voltage-clamp was used to voltage-clamp potassium currents in standard Ringers solution (in $\mathrm{mM}$ ): $116 \mathrm{NaCl}$, $2 \mathrm{KCl}, 1 \mathrm{MgCl}_{2}, 0.5 \mathrm{CaCl}_{2}, 5 \mathrm{HEPES}(\mathrm{pH} 7.4)$ using an OC-725C voltage clamp (Warner, Hamden, CT). Glass microelectrodes were backfilled with $3 \mathrm{M} \mathrm{KCl}$ and had resistances of $0.1-1 \mathrm{M} \Omega$. Typically, the holding potential was $-80 \mathrm{mV}$ and depolarizing voltage-pulses were given for $50-500 \mathrm{~ms}$ in 5 and $20 \mathrm{mV}$ increments for conventional two electrode voltage-clamp and VCF, respectively. For VCF experiments, oocytes were labelled on ice in a depolarizing solution containing (in $\mathrm{mM}$ ) $100 \mathrm{KCl}, 1.5 \mathrm{MgCl}_{2}, 0.5 \mathrm{CaCl}_{2}, 10 \mathrm{HEPES}(\mathrm{pH} 7.4$ ) and $10 \mu \mathrm{M}$ tetramethylrhodamine-5-maleimide (tetramethylrhodamine-5-maleimide; Invitrogen, Carlsbad, CA) for 20-30 min. Oocytes were subsequently washed and stored in Ringers solution for up to $2 \mathrm{~h}$ on ice. The VCF set up consisted of an Olympus IX51 inverted microscope, a PMT400 photomultiplier tube (IonOptix, Milton, MA), a XCite 120 Q light source (Lumen Dynamics Group, Mississauga, ON, Canada) and a Lambda SC shutter to minimize photo bleaching (Sutter Instruments, Novato, CA). The fluorescence signal was acquired with a sampling frequency of $1 \mathrm{kHz}$ and 20 50 sweeps were averaged for analysis; for display, fluorescence traces were offline filtered at 300-800 Hz. Conductance-voltage (GV) relationships were obtained by plotting the isochronal tail current amplitudes against the depolarizing pulse potential or by dividing the measured current amplitude at a given voltage by the driving force (assuming a potassium reversal potential of $-84 \mathrm{mV}$ ). The peak fluorescence signals were plotted as a function of the depolarizing pulse potential to obtain fluorescence-voltage relationships. All data $=$ mean \pm s.e.m.

Kinetic modelling. The Zagotta-Hoshi-Aldrich model of Shaker gating was employed to model the effects of manipulating different rate constants ${ }^{29}$. IonChannelLab 1.0.5.3 was used to run the model and to simulate both steady-state curves and deactivation kinetics ${ }^{58}$. Deactivation time constants of the simulated 
data were fitted with single exponentials in Clampfit 10.2.0.12 (Molecular Devices, Sunnyvale, CA).

SCA and $\boldsymbol{a b}$ initio computations. SCA ${ }^{35}$ was performed based on a multiple sequence alignment (MSA) representing 360 members of the Kv family, as used previously ${ }^{37}$. We used multiple iterations of a PSI-BLAST $(e$-score $<0.001)$ on a non-redundant database to generate the MSA, and used KvAP, Shaker and BK channel sequences for the initial query ${ }^{59}$. Only the sequence regions of the voltagesensor and/or pore regions were used; further details of the MSA are described elsewhere ${ }^{37}$. SCA was performed using the SCA 5 MATLAB toolbox downloaded from the Ranganathan Lab website (http://systems.swmed.edu/rr_lab), deployed in MATLAB Ver. 7.10 (MathWorks, Natick, MA). Instead of truncating the MSA based on gap-frequency, we used the whole alignment and then refined the data to only include those positions found on the Shaker sequence for analysis and figures. $A b$ initio computations gas phase Hartree-Fock 6-31G calculations were performed using Gaussian09/GaussView 5.0 (Gaussian, Wallingford, CT) as described elsewhere ${ }^{57}$

\section{References}

1. Hille, B. Ion channels of excitable membranes. 3rd edn (Sinauer, 2001).

2. Jiang, Y. et al. The open pore conformation of potassium channels. Nature 417, 523-526 (2002).

3. Long, S. B., Tao, X., Campbell, E. B. \& MacKinnon, R. Atomic structure of a voltage-dependent $\mathrm{K}+$ channel in a lipid membrane-like environment. Nature 450, 376-382 (2007).

4. Jan, L. Y. \& Jan, Y. N. Voltage-gated potassium channels and the diversity of electrical signalling. J. Physiol. 590, 2591-2599 (2012).

5. Nerbonne, J. M. \& Kass, R. S. Molecular physiology of cardiac repolarization. Physiol. Rev. 85, 1205-1253 (2005).

6. Wulff, H., Castle, N. A. \& Pardo, L. A. Voltage-gated potassium channels as therapeutic targets. Nat. Rev. Drug Discov. 8, 982-1001 (2009).

7. Yifrach, O. \& MacKinnon, R. Energetics of pore opening in a voltage-gated $\mathrm{K}(+)$ channel. Cell 111, 231-239 (2002).

8. Jensen, M. O. et al. Principles of conduction and hydrophobic gating in $\mathrm{K}+$ channels. Proc. Natl Acad. Sci. USA. 107, 5833-5838 (2010).

9. Jensen, M. O. et al. Mechanism of voltage gating in potassium channels. Science 336, 229-233 (2012).

10. Kanevsky, M. \& Aldrich, R. W. Determinants of voltage-dependent gating and open-state stability in the S5 segment of Shaker potassium channels. J. Gen. Physiol. 114, 215-242 (1999).

11. Li-Smerin, Y., Hackos, D. H. \& Swartz, K. J. A localized interaction surface for voltage-sensing domains on the pore domain of a $\mathrm{K}+$ channel. Neuron 25, 411-423 (2000).

12. Soler-Llavina, G. J., Chang, T. H. \& Swartz, K. J. Functional interactions at the interface between voltage-sensing and pore domains in the Shaker K(v) channel. Neuron 52, 623-634 (2006).

13. Ding, S. \& Horn, R. Effect of S6 tail mutations on charge movement in Shaker potassium channels. Biophys. J. 84, 295-305 (2003).

14. Ferrer, T., Rupp, J., Piper, D. R. \& Tristani-Firouzi, M. The S4-S5 linker directly couples voltage sensor movement to the activation gate in the human ether-a'go-go-related gene (hERG) K + channel. J. Biol. Chem. 281, 12858-12864 (2006).

15. Labro, A. J. et al. Kv channel gating requires a compatible S4-S5 linker and bottom part of S6, constrained by non-interacting residues. J. Gen. Physiol. 132, 667-680 (2008).

16. Lu, Z., Klem, A. M. \& Ramu, Y. Ion conduction pore is conserved among potassium channels. Nature 413, 809-813 (2001).

17. Lu, Z., Klem, A. M. \& Ramu, Y. Coupling between voltage sensors and activation gate in voltage-gated $\mathrm{K}+$ channels. J. Gen. Physiol. 120, 663-676 (2002).

18. Tristani-Firouzi, M., Chen, J. \& Sanguinetti, M. C. Interactions between S4-S5 linker and S6 transmembrane domain modulate gating of HERG $\mathrm{K}+$ channels. J. Biol. Chem. 277, 18994-19000 (2002).

19. Batulan, Z., Haddad, G. A. \& Blunck, R. An intersubunit interaction between S4-S5 linker and S6 is responsible for the slow off-gating component in Shaker K + channels. J. Biol. Chem. 285, 14005-14019 (2010).

20. Boulet, I. R., Labro, A. J., Raes, A. L. \& Snyders, D. J. Role of the S6 C-terminus in KCNQ1 channel gating. J. Physiol. 585, 325-337 (2007).

21. Labro, A. J. et al. The S4-S5 linker of KCNQ1 channels forms a structural scaffold with the S6 segment controlling gate closure. J. Biol. Chem. 286 717-725 (2011)

22. Nowak, M. W. et al. In vivo incorporation of unnatural amino acids into ion channels in Xenopus oocyte expression system. Methods Enzymol. 293, 504-529 (1998).

23. Hackos, D. H., Chang, T. H. \& Swartz, K. J. Scanning the intracellular S6 activation gate in the shaker K + channel. J. Gen. Physiol. 119, 521-532 (2002).

24. Burley, S. K. \& Petsko, G. A. Amino-aromatic interactions in proteins. FEBS Lett. 203, 139-143 (1986).
25. Dougherty, D. A. Cation-pi interactions in chemistry and biology: a new view of benzene, Phe, Tyr, and Trp. Science 271, 163-168 (1996).

26. Zhong, W. et al. From ab initio quantum mechanics to molecular neurobiology: a cation-pi binding site in the nicotinic receptor. Proc. Natl Acad. Sci. USA. 95, 12088-12093 (1998).

27. Prole, D. L. \& Yellen, G. Reversal of HCN channel voltage dependence via bridging of the S4-S5 linker and Post-S6. J. Gen. Physiol. 128, 273-282 (2006).

28. Haddad, G. A. \& Blunck, R. Mode shift of the voltage sensors in Shaker K+ channels is caused by energetic coupling to the pore domain. J. Gen. Physiol. 137, 455-472 (2011).

29. Zagotta, W. N., Hoshi, T. \& Aldrich, R. W. Shaker potassium channel gating III: Evaluation of kinetic models for activation. J. Gen. Physiol. 103, 321-362 (1994).

30. Ledwell, J. L. \& Aldrich, R. W. Mutations in the S4 region isolate the final voltage-dependent cooperative step in potassium channel activation. J. Gen. Physiol. 113, 389-414 (1999).

31. Smith-Maxwell, C. J., Ledwell, J. L. \& Aldrich, R. W. Uncharged S4 residues and cooperativity in voltage-dependent potassium channel activation. J. Gen. Physiol. 111, 421-439 (1998).

32. Kurata, H. T. \& Fedida, D. A structural interpretation of voltage-gated potassium channel inactivation. Prog. Biophys. Mol. Biol. 92, 185-208 (2006).

33. Cha, A. \& Bezanilla, F. Characterizing voltage-dependent conformational changes in the Shaker K + channel with fluorescence. Neuron 19, 1127-1140 (1997).

34. Mannuzzu, L. M., Moronne, M. M. \& Isacoff, E. Y. Direct physical measure of conformational rearrangement underlying potassium channel gating. Science 271, 213-216 (1996).

35. Suel, G. M., Lockless, S. W., Wall, M. A. \& Ranganathan, R. Evolutionarily conserved networks of residues mediate allosteric communication in proteins. Nat. Struct. Biol. 10, 59-69 (2003).

36. Lockless, S. W. \& Ranganathan, R. Evolutionarily conserved pathways of energetic connectivity in protein families. Science 286, 295-299 (1999).

37. Lee, S. Y., Banerjee, A. \& MacKinnon, R. Two separate interfaces between the voltage sensor and pore are required for the function of voltage-dependent $\mathrm{K}(+)$ channels. PLoS. Biol 7, e47 (2009).

38. Papazian, D. M., Timpe, L. C., Jan, Y. N. \& Jan, L. Y. Alteration of voltagedependence of Shaker potassium channel by mutations in the S4 sequence. Nature 349, 305-310 (1991)

39. Perozo, E., Santacruz-Toloza, L., Stefani, E., Bezanilla, F. \& Papazian, D. M. S4 mutations alter gating currents of Shaker K channels. Biophys. J. 66, 345-354 (1994).

40. Gallivan, J. P. \& Dougherty, D. A. Cation-pi interactions in structural biology. Proc. Natl Acad. Sci. USA. 96, 9459-9464 (1999).

41. Pless, S. A., Galpin, J. D., Niciforovic, A. P. \& Ahern, C. A. Contributions of counter-charge in a potassium channel voltage-sensor domain. Nat. Chem. Biol. 7, 617-623 (2011).

42. Pathak, M. M. et al. Closing in on the resting state of the Shaker $\mathrm{K}(+)$ channel. Neuron 56, 124-140 (2007).

43. Chen, X., Wang, Q., Ni, F. \& Ma, J. Structure of the full-length Shaker potassium channel Kv1.2 by normal-mode-based X-ray crystallographic refinement. Proc. Natl Acad. Sci. USA. 107, 11352-11357 (2010).

44. Dawson, R. E. et al. Experimental evidence for the functional relevance of anion-pi interactions. Nat. Chem. 2, 533-538 (2010).

45. Lampert, A. et al. A pore-blocking hydrophobic motif at the cytoplasmic aperture of the closed-state Nav1.7 channel is disrupted by the erythromelalgiaassociated F1449V mutation. J. Biol. Chem. 283, 24118-24127 (2008).

46. Dib-Haji, S. D. et al. Gain-of-function mutation in Nav1.7 in familial erythromelalgia induces bursting of sensory neurons. Brain 128, 1847-1854 (2005).

47. Boland, L. M., Price, D. L. \& Jackson, K. A. Episodic ataxia/myokymia mutations functionally expressed in the Shaker potassium channel. Neuroscience 91, 1557-1564 (1999).

48. Uysal, S. et al. Mechanism of activation gating in the full-length KcsA K+ channel. Proc. Natl Acad. Sci. USA 108, 11896-11899 (2011).

49. Uysal, S. et al. Crystal structure of full-length KcsA in its closed conformation. Proc. Natl Acad. Sci. USA 106, 6644-6649 (2009).

50. Schottel, B. L., Chifotides, H. T. \& Dunbar, K. R. Anion-pi interactions. Chem. Soc. Rev. 37, 68-83 (2008).

51. Santarelli, V. P., Eastwood, A. L., Dougherty, D. A., Ahern, C. A. \& Horn, R. Calcium block of single sodium channels: role of a pore-lining aromatic residue. Biophys. J. 93, 2341-2349 (2007).

52. Isom, D. G., Cannon, B. R., Castaneda, C. A., Robinson, A. \& Garcia-Moreno, B. High tolerance for ionizable residues in the hydrophobic interior of proteins. Proc. Natl Acad. Sci. USA. 105, 17784-17788 (2008).

53. Liu, L. et al. A glutamate switch controls voltage-sensitive phosphatase function. Nat. Struct. Mol. Biol. 19, 633-641 (2012). 
54. Yerushalmi, H. \& Schuldiner, S. An essential glutamyl residue in EmrE, a multidrug antiporter from Escherichia coli. J. Biol. Chem. 275, 5264-5269 (2000).

55. Liu, L., Baase, W. A., Michael, M. M. \& Matthews, B. W. Use of stabilizing mutations to engineer a charged group within a ligand-binding hydrophobic cavity in T4 lysozyme. Biochemistry 48, 8842-8851 (2009).

56. Shoichet, B. K., Baase, W. A., Kuroki, R. \& Matthews, B. W. A relationship between protein stability and protein function. Proc. Natl Acad. Sci. USA. 92, 452-456 (1995)

57. Pless, S. A., Galpin, J. D., Frankel, A. \& Ahern, C. A. Molecular basis for class Ib anti-arrhythmic inhibition of cardiac sodium channels. Nat. Commun. 2, 351 (2011).

58. Santiago-Castillo, J. A., Covarrubias, M., Sanchez-Rodriguez, J. E., Perez Cornejo, P. \& Arreola, J. Simulating complex ion channel kinetics with IonChannelLab. Channels 4, 422-428 (2010).

59. Altschul, S. F. et al. Gapped BLAST and PSI-BLAST: a new generation of protein database search programs. Nucleic Acids Res. 25, 3389-3402 (1997).

\section{Acknowledgements}

This work was supported by the Canadian Institutes of Health Research (MOP-56858, MOP-97998), the Heart and Stroke Foundation of Canada (to C.A.A., H.T.K.), the Michael Smith Foundation for Health Research (to C.A.A., H.T.K. and S.A.P.), a Banting postdoctoral fellowship by the Canadian Institutes of Health Research (to S.A.P.), and a NSERC Undergraduate Student Research Award (to J.J.N.). C.A.A is a member of the
Membrane Protein Structural Dynamics Consortium, which is funded by the NIH/ NIGMS grant no. GM087519.

\section{Author contributions}

S.A.P. performed and analysed the experiments, A.P.N. provided molecular biology support, J.D.G. synthesized chemical reagents and J.J.N. conducted statistical coupling analysis and kinetic modelling. S.A.P., H.T.K. and C.A.A. designed the research and prepared the manuscript.

\section{Additional information}

Supplementary Information accompanies this paper at http://www.nature.com/ naturecommunications

Competing financial interests: The authors declare no competing financial interests.

Reprints and permission information is available online at http://npg.nature.com/ reprintsandpermissions/

How to cite this article: Pless, S. A. et al. A novel mechanism for fine-tuning open-state stability in a voltage-gated potassium channel. Nat. Commun. 4:1784 doi: 10.1038/ ncomms2761 (2013).

This work is licensed under a Creative Commons AttributionNonCommercial-No Derivs 3.0 Unported License. To view a copy of this license, visit http://creativecommons.org/licenses/by-nc-sa/3.0/ 